\title{
Hippocampal volume reduction is associated with intellectual functions in adolescents with congenital heart disease
}

\author{
Beatrice Latal ${ }^{1,2}$, Pratik Patel ${ }^{1}$, Rabia Liamlahi', Walter Knirsch ${ }^{2,3}$, Ruth O'Gorman Tuura ${ }^{2,4}$, Michael von Rhein ${ }^{1,2,5}$; on behalf of the \\ Research Group Heart and Brain.
}

BACKGROUND: Adolescents undergoing early cardiopulmonary bypass surgery for congenital heart disease (CHD) may demonstrate a variety of neurocognitive impairments. These impairments can affect overall intellectual functions, but also specific memory deficits, language, and executive functions. As the hippocampus is a critical structure involved in these functions, we sought to determine whether hippocampal volume was reduced in adolescents with CHD and whether altered volumes were related to functional outcome.

METHODS: At a mean age of 13.8 y, 48 adolescent survivors of childhood cardiopulmonary bypass surgery for CHD and 32 healthy controls underwent neurocognitive testing and cerebral magnetic resonance imaging. Images were quantitatively analyzed using an automated regional segmentation tool (FSL-FIRST). RESULTS: Adolescents with CHD had 10\% lower total hippocampal volumes compared with controls. After controlling for total brain volume, total hippocampal volume correlated with total IQ, with working memory, and verbal comprehension in CHD patients, but not in controls.

CONCLUSIONS: In adolescent survivors of cardiopulmonary bypass surgery for $\mathrm{CHD}$, specific brain regions such as the hippocampus may show long-term persistent alteration and correlate with intellectual deficits, particularly with verbal and memory functions.

$I^{n}$ n children with congenital heart disease (CHD), long-term neurodevelopmental impairments may occur. Problems include overall intellectual deficits, learning difficulties, language problems as well as visuospatial, visuomotor, and executive dysfunctions (1). There is evidence that these difficulties may persist into adolescence in patients with corrected transposition of the great arteries (2) and other forms of CHD $(3,4)$.

The underlying etiology is not completely understood, but delayed brain development as well as pre- and postoperative brain injury can occur in infants undergoing open-heart surgery for $\mathrm{CHD}$ (5-8). Intrauterine brain growth decelerates in fetuses with complex forms of CHD, starting to diverge from expected growth curves at around $30 \mathrm{wk}$ of gestation (7).
Furthermore, the patterns of brain injury found preoperatively in CHD neonates appear similar to the one found in preterm neonates (5). After birth, brain volumes can be reduced preoperatively $(8,9)$ as well as postoperatively $(8)$. Magnetic resonance imaging (MRI) studies in adolescents with CHD have shown that there may be a persistence of cerebral alterations $(2,10)$ with a reduction of global brain volume also in the absence of overt brain lesions (10) and altered white matter microstructure (11).

The hippocampus is a crucial brain structure involved in memory functions and overall intellectual performance (12). Associations between hippocampal volumes and learning difficulties have been demonstrated in preterm born children $(13,14)$. We thus sought to determine whether hippocampal volumes were particularly reduced in adolescents after bypass cardiopulmonary surgery for CHD and whether altered volumes would be related to functional outcomes. Our hypothesis was that hippocampal volumes would be reduced in adolescents with CHD and that hippocampal volumes would be related to intellectual outcome, in particular to working and spatial memory.

\section{RESULTS}

\section{Patient Characteristics}

Medical characteristics of the patient group are shown in Table 1. No significant differences of medical characteristics were found between patients with overt anatomical lesions $(n=9)$ and patients without lesions $(n=39)$. Mean full scale IQ of CHD patients was normal, but lower than in controls (mean 103.1, SD 16.5 vs. 112.7, SD 10.4; $P=0.0001$ ). Also, performance in perceptual reasoning (103.7, SD 14.8 vs. 113.8, SD 8.8; $P<0.001)$ and working memory $(93.4$, SD 13.6 vs. 103.8 , SD 12.2; $P<0.001)$ was poorer in CHD patients, whereas verbal comprehension (109.1, SD 18.8 vs. 115.2, SD 16.0; $P=0.1$ ) and processing speed (101.5, SD 14.2 vs. 105.4, SD 11.2; $P=0.1$ ) did not differ significantly between the groups. No significant difference in outcome was found between patients with cyanotic and acyanotic CHD. For detailed neurocognitive outcome of this population, see refs. $(3,15)$.

\footnotetext{
'Child Development Center, University Children's Hospital, Zurich, Switzerland; ${ }^{2}$ Children's Research Center, University Children's Hospital, Zurich, Switzerland; ${ }^{3}$ Division of Paediatric Cardiology, Paediatric Heart Center, University Children's Hospital, Zurich, Switzerland; ${ }^{4}$ MR-Center, University Children's Hospital, Zurich, Switzerland; ${ }_{5}^{5}$ Developmental Pediatrics, Kantonsspital, Winterthur, Switzerland. Correspondence: Beatrice Latal (Bea.Latal@kispi.uzh.ch) 


\section{Articles | Lataletal.}

Table 1. Characteristics of adolescents with $\operatorname{CHD}(n=48)$

\begin{tabular}{lc}
\hline Male sex & $21(44)$ \\
Premature birth (<37 wk of gestation) $(n / \%)$ & $8(17)$ \\
Birth weight $(\mathrm{g})$ & $3,090(940-4,370)$ \\
Type of CHD & $15(31)$ \\
ASD/VSD & $9(19)$ \\
Other non-cyanotic & $9(19)$ \\
TGA & $15(31)$ \\
Other cyanotic & $8.3(7-10)$ \\
5 Apgar & $1.4(0-5.6)$ \\
Age at first surgery (y) & $8.3(2.8-19.5)$ \\
Weight at surgery (kg) & $91(15-162)$ \\
Bypass time (min) & $7(15)$ \\
EEG/focal neurological findings postsurgery & $1.3(1-4)$ \\
Total number of surgeries & \\
\hline Median (range) $N$ (\%). & \\
ASD, atrial septum defect; CHD, congenital heart disease;TGA, transposition of the \\
great arteries; VSD, ventricle septum defect.
\end{tabular}
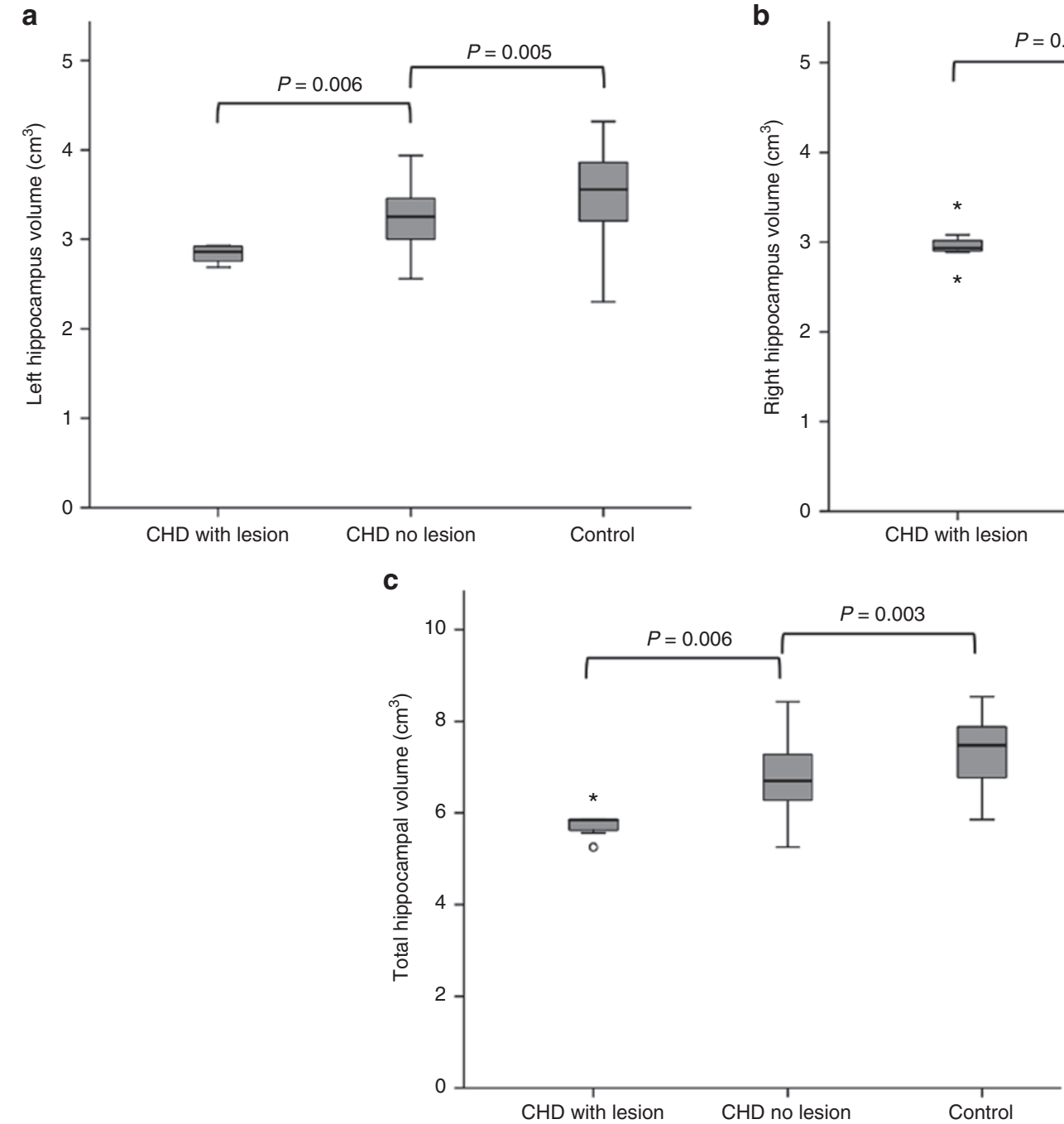

Figure 1. Hippocampal volumes of controls and CHD patients (with and without cerebral lesions). (a) left hippocampal volume, (b) right hippocampal volume, (c) total hippocampal volume, $P$ : two-sided $t$-tests between controls, and CHD patients without overt lesions, and CHD patients with and without lesions, respectively. CHD, congenital heart disease.

\section{Anatomic Abnormalities}

Cerebral lesions were detected in 9 of the 48 patients reported in this study (15), consisting of mild white matter changes, poststroke changes, and periventricular volume loss with ventricular enlargement. Total brain volume was $8.5 \%$ smaller in $\mathrm{CHD}$ patients compared with age-matched controls (see ref. (10)).

\section{Hippocampal Volumes}

Adolescents with $\mathrm{CHD}$ had lower total hippocampal volumes (9.6\%, $P=0.003)$ compared with controls. Also, both right and patients compared with controls, and significantly smaller in CHD patients with lesions $(n=9)$ compared with patients without lesions $(n=39)$ on conventional MRI (Figure 1). After exclusion of patients with overt cerebral lesions, differences in hippocampal volumes remained significant. When calculating relative hippocampal volumes (expressed as \% of total brain volume), volumes in CHD patients were similar to those in controls $(0.72 \%$ vs. $0.73 \%$ of total brain volume, $P=0.34)$. Both

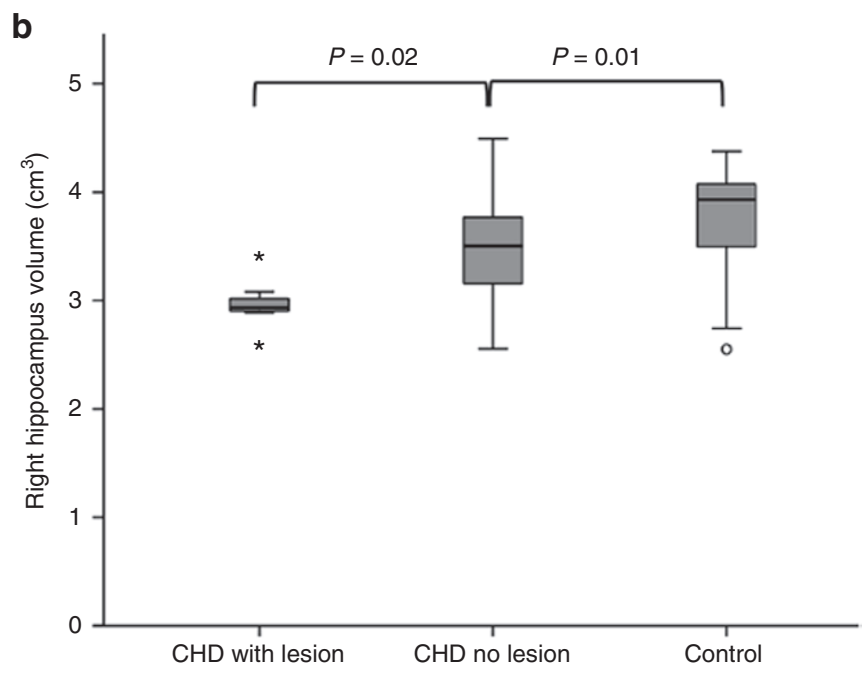
left hippocampal volumes were significantly smaller in CHD 
groups showed hippocampal asymmetry with a larger right hippocampus, but differences were neither significant in CHD patients nor in controls.

We also looked at a subgroup of patients $(n=19)$ who were operated prior to 9 mo of age. As in the entire cohort, hippocampal volumes were significantly different between CHD patients $(6.6 \mathrm{ml} \mathrm{SD} \mathrm{0.7)}$ and controls (7.3 ml SD 0.8) within this subgroup $(P=0.003)$. In addition, hippocampal volume between those operated prior to 9 mo of age and those older than 9 mo of age was not different $(7.2 \%$ vs. $7.3 \%$ of total brain volume, $P=0.2)$.

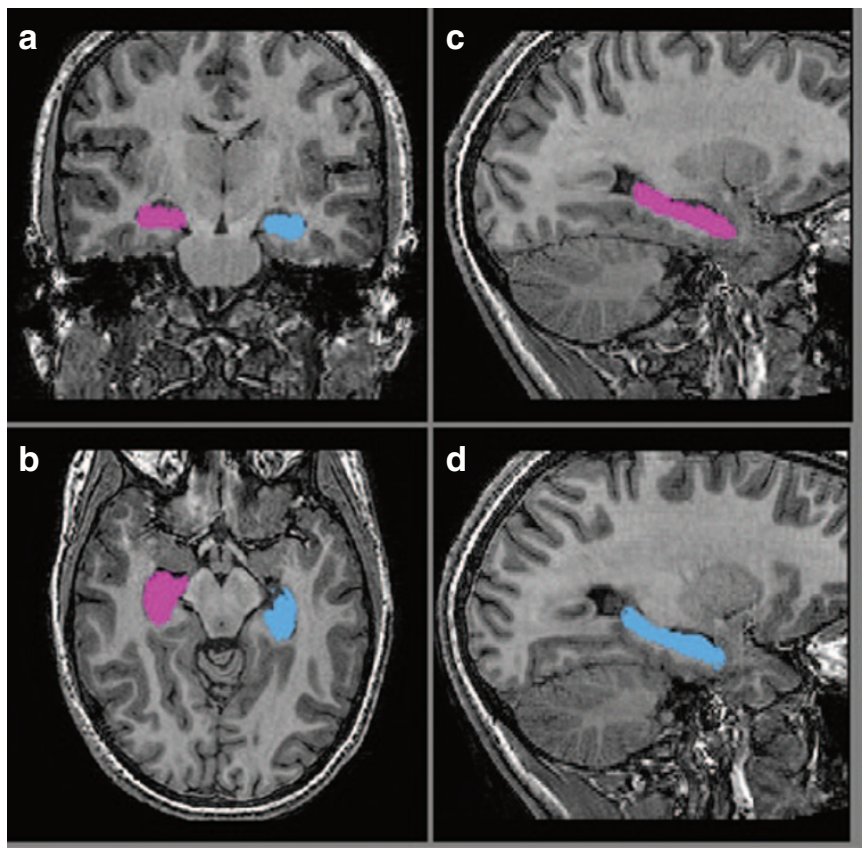

Figure 2. Example of hippocampal segmentation. Delineation of the right (blue) and left (purple) hippocampus based on FIRST segmentations in (a) coronal, (b) axial, and (c,d) sagittal plains.

a

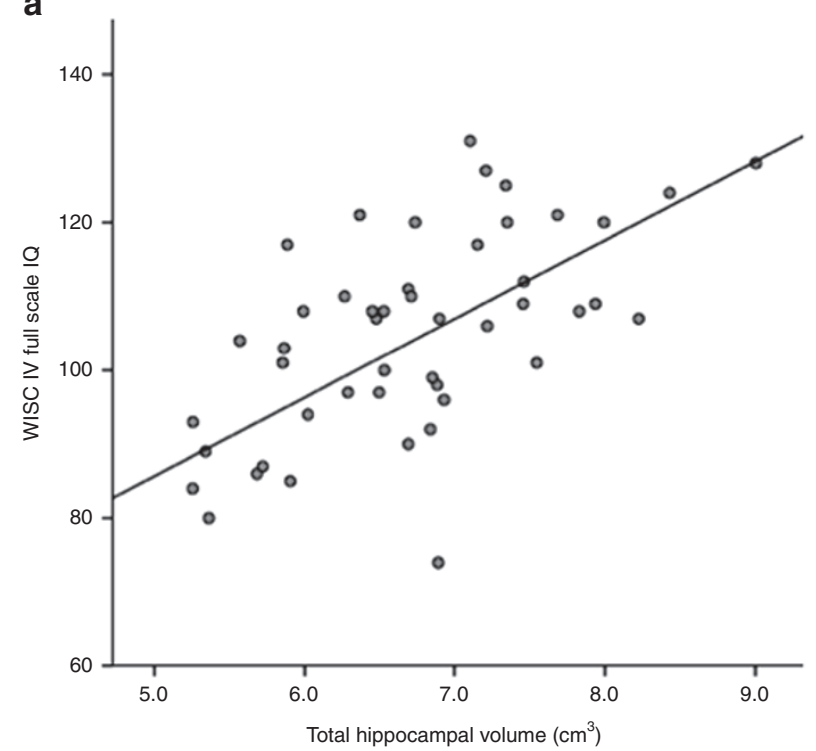

\section{Correlation Between Hippocampal Volume and Functional} Outcome

After exclusion of adolescents with overt brain lesions $(n=9)$, hippocampal volume correlated significantly with total IQ in CHD patients (Spearman's rho $=0.61, P<0.001$; Figure 3 ), but not in controls (Spearman's rho $=0.09, P=0.60$ ). Significant correlations were also found between hippocampal volume and verbal comprehension (Spearman's rho $=0.59, P<0.001$ ), and working memory (Spearman's rho $=0.36, P=0.01$ ), respectively. Regression analyses showed that these associations in CHD patients were independent of age, gender, and total brain volume (Table 2). Hippocampal volume was neither correlated with processing speed nor with perceptual reasoning. No correlation was found between hippocampal volume and performance on the Beery Test and on the Rey Figure.

\section{Risk Factors}

A cyanotic CHD was not associated with smaller hippocampal volume compared with acyanotic CHD (6.7, SD 0.8 vs. 7.0, SD $\left.0.8 \mathrm{~cm}^{3}, P=0.10\right)$. No other potential clinical risk factor (age at surgery, socioeconomic status, duration of cardiopulmonary bypass surgery, number of surgeries, length of hospital stay, or postoperative neurological abnormalities) correlated with hippocampal volume.

\section{DISCUSSION}

In this study, we could demonstrate that hippocampal volumes were significantly reduced in adolescents after cardiopulmonary bypass surgery for CHD compared with age-matched healthy controls. The decrease in volume was more pronounced in adolescents with cerebral lesions. Hippocampal volume reduction showed a significant association with poorer intellectual performance, and poorer memory and verbal functions. Importantly, the correlation was independent of total brain volume, while hippocampal volume reduction in

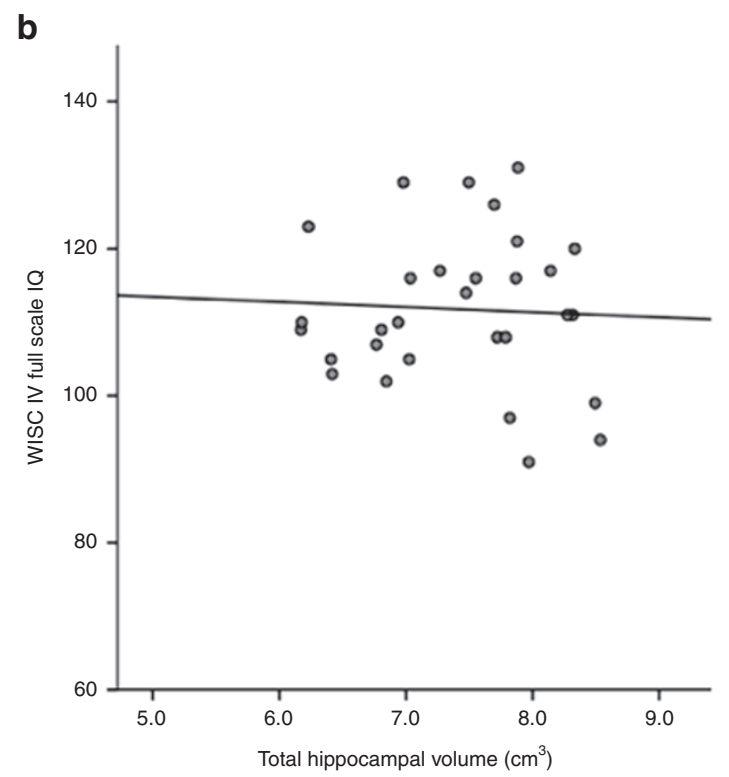

Figure 3. Scatterplot of hippocampal volume versus total IQ, plotted separately for CHD patients and controls. (a) CHD patients without brain lesions, Spearman's rho $=0.607, P<0.001$; (b) control group, Spearman's rho $=0.094, P=0.6$. CHD, congenital heart disease. 
Table 2. Total hippocampal volume in relation to intellectual outcome stratified for CHD and controls

\begin{tabular}{lcccccccc}
\hline & \multicolumn{3}{c}{$\mathrm{CHD}, N=39$} & & \multicolumn{3}{c}{ Control, $N=32$} \\
\cline { 2 - 4 } \cline { 7 - 9 } & $\begin{array}{c}\text { Corrected } \\
R^{2}\end{array}$ & Beta & value & \multicolumn{1}{c}{$R^{2}$} & Beta & $P$ value \\
\hline Total IQ & 0.32 & 0.58 & 0.003 & -0.07 & -0.15 & 0.5 \\
Verbal & 0.22 & 0.57 & 0.006 & 0.06 & -0.35 & 0.1 \\
comprehension & & & & & & \\
Perceptual reasoning & 0.07 & 0.35 & 0.1 & -0.01 & 0.21 & 0.3 \\
Working memory & 0.16 & 0.45 & 0.03 & 0.06 & -0.22 & 0.3 \\
Processing speed & 0.18 & 0.28 & 0.2 & -0.07 & -0.01 & 0.9 \\
\hline
\end{tabular}

$C H D$, congenital heart disease; $\mathrm{Q}$, intelligence quotient.

Linear regression analyses (dependent variable: functional outcomes). Variables included: total hippocampal volume, total brain volume, sex, and age at scan (patients with brain lesions excluded).

CHD adolescents was not specifically affected compared with overall brain volume reduction. In contrast, in healthy controls, hippocampal volume did not correlate with intellectual performance. We therefore conclude that the hippocampus is particularly affected in children with CHD and correlates with functional deficits. As we did not include patients with severe neurocognitive impairments, the findings cannot be explained by a small group of severely affected patients.

It has been shown that neurocognitive deficits may persist into adolescence in CHD patients $(2,16-18)$, and these deficits correlate with global brain volume (10) and with alterations in white matter network topology (11). The spectrum of deficits, consisting of reduced overall IQ, specific learning difficulties (language, reading, mathematics), and deficits in executive functions, is very similar to those found in preterm born adolescents (19), in whom the hippocampus has been identified as a particularly vulnerable structure $(20,21)$. In a study by Peterson et al., examining 25 preterm children at the age of $8 \mathrm{y}$, a variety of regions showed volumetric alterations, in particular the cerebellum, basal ganglia, the amygdala, and both hippocampi, which were also more reduced in size than expected from the overall brain volume reduction. They found volumetric differences of $12 \%$ and $16 \%$ in the right and left hippocampus, respectively (22). This difference is slightly higher than the approximately $10 \%$ found in our population of CHD adolescents. Interestingly, in their study, hippocampal volume did not independently correlate with overall IQ or other subscales whereas cortical regions did.

Our study is the first to show similar findings for adolescent survivors of childhood cardiac surgery as described for the preterm population, supporting the hypothesis that these two populations may share timing and mechanisms of brain injury and therefore present with similar clinical and neuroanatomic long-term findings (23).

The hippocampus is involved in various higher cognitive functions (24) such as fluid intelligence (25) and different aspects of memory including working memory (26-28), but also declarative/episodic memory, constructive memory, and spatial navigation $(29,30)$. Beauchamp et al. found that hippocampal volumes in preterm born infants assessed at term equivalent age correlated with working memory deficits at the age of 2 y (13). Harms et al. (31) reported a structure-function relationship between hippocampal and prefrontal volumes with working memory activity, suggesting that the hippocampus is an important component of the working memory system, as part of a distributed network also including prefrontal and parietal regions $(32,33)$. In future studies it will be important to examine functional and structural changes in both the prefrontal cortex and the hippocampus in relation to working memory alterations in children with CHD. The hippocampus is a very plastic structure with high-energy demands and is particularly vulnerable to injurious effects, such as hypoxicischemic injury, around the time of birth (34). It is conceivable that the variability in hippocampal size in healthy controls does not explain much of the variance in functional performance, as in healthy adolescents size and function represent the variability of the normal spectrum. It seems that interindividual variations in the number or size of neurons, neurogenesis, or connectivity depending on dendritic and axonal arborization and the number of synapses leads to a wide variability of hippocampal size, which does not necessarily correspond to functional performance (35). In contrast, some studies even found a significant negative correlation of hippocampal size and memory in healthy children, adolescents, and young adults (36). Nevertheless, it is widely recognized that a loss in hippocampal volume can be associated with impairments in neurocognitive function after brain injury or developmental alteration (36). It is conceivable that the hippocampus may represent a particularly vulnerable structure in children with $\mathrm{CHD}$, which may explain the correlation with neurocognitive function. We could show that correlations between hippocampal size and functional outcome were independent of overall brain volume reduction in CHD patients (10).

Importantly, we could not determine the timing of hippocampal injury in our study as we only examined adolescents with CHD and no neonatal MRI was obtained. As the effects of delayed fetal brain maturation are probably best seen in those children with the most severe CHD who undergo surgery during infancy, we also performed a subgroup analysis for those infants who were operated prior to 9 mo of age (37). We found that, similar to the analysis of the entire cohort, hippocampal volumes were significantly different between patients and controls. Interestingly, hippocampal volume did not correlate with functional outcome in this subsample, probably due to smaller sample size. In addition, hippocampal volume was similar between the groups of children operated before or after 9 mo of age. This finding indicates that children with CHD are at risk for long-term adverse effects on hippocampal volume and function, and this effect seems independent of the time of surgery. Currently, there are no published studies examining the relationship between hippocampal size in the neonatal period and neurodevelopmental outcome in CHD patients. However, in preterm born infants who underwent cerebral MRI at term-equivalent age, hippocampal volumes correlated with working memory deficits at $2 \mathrm{y}$ of age (13) and with socioemotional behavior at $5 \mathrm{y}$ of age in females (38). To confirm 
such a relationship in cardiac patients would be important as it would support the findings of our study in older patients with $\mathrm{CHD}$ and would help to improve outcome prediction.

\section{Limitations}

Our study has limitations worth mentioning. We included a broad spectrum of CHD diagnoses and also included those with milder forms of CHD who were older than $1 \mathrm{y}$ when they underwent cardiopulmonary bypass surgery. Thus, the group of patients with newborn surgery was too small to analyze separately. As we did not have fetal or neonatal MRI scans in our population, we were not able to specify the evolution of altered brain development leading to the smaller hippocampal volume in adolescence. We included different types of CHD, but the sample size was too small to examine whether our findings would hold true for all subtypes of CHD's. Consequently, we were limited in the analysis of risk factors for reduced hippocampal volume. We therefore may have underestimated contributing surgical factors or other risk factors. As we examined adolescents with CHD, we were unable to determine the timing and mechanisms of altered hippocampal volumes. Also, we did not perform extended assessment of memory function and thus may have missed certain aspects of memory function that might have shown a correlation with hippocampal volume.

\section{Conclusion}

In adolescent survivors of childhood cardiopulmonary bypass surgery for CHD, specific brain regions such as the hippocampus show persistent volume reductions that correlate with poorer intellectual performance, particularly with verbal and memory functions. The timing and mechanisms leading to these specific brain alterations need to be studied to improve long-term outcome for these patients.

\section{METHODS}

\section{Participants}

Eligible adolescents underwent full-flow cardiopulmonary bypass surgery for a broad spectrum of CHD diagnoses at the University Children's Hospital Zurich. Surgeries took place between 1995 and 1998 (median age at first surgery: $0.9 \mathrm{y}$; range $0-5.6 \mathrm{y}$ ). We recruited adolescents from a former cross-sectional study on 117 children who were examined at a mean age of $10 \mathrm{y}$ to determine neurodevelopmental outcome, psychological adaptation, and health related quality of life at school age (39). Adolescents with an underlying genetic disorder, who were older than $16 \mathrm{y}$ at the time of this study, who were wearing braces, pacemakers, or cochlear implants were excluded. Of 76 eligible adolescents, 23 either refused participation or were lost to follow-up. Thus, 53 adolescents were examined at a median age of $13.8 \mathrm{y}$ (range 11.4-16.9, 46\% male). Demographic and surgical characteristics were not significantly different between participants and patients lost to follow-up. Morphometric measurements could be analyzed in 48 patients, as MRI data from 5 patients were excluded due to poor image quality. Results were compared with 32 healthy age- and sexmatched controls (median age $13.9 \mathrm{y}$, range 9-16.9; 41\% male). All controls went to regular school and none suffered any chronic or neurological diseases. None of the controls had brain lesions on MRI. The groups were matched for socioeconomic status and underwent identical neurodevelopmental assessment.

Ethical approval was obtained from the Ethical Commission of the University Children's Hospital, and written informed consent was obtained from the parent or primary caregiver and from the adolescent.

\section{Neurocognitive Outcome}

Intellectual outcome was assessed with the Wechsler Intelligence Scale for Children 4th edition. Further, the Beery Test of visuomotor integration and the Rey-Osterrieth Complex Figure Test were administered. A detailed description of neurocognitive outcome assessment tools were reported previously (15).

For the current analysis, the full scale IQ and the four Wechsler Intelligence Scale for Children subscales (verbal comprehension, perceptual reasoning, working memory, and processing speed) were analyzed. The neurodevelopmental assessment was performed prior to the MRI examination by an experienced pediatric neurologist (MvR) who was aware of the medical condition but not the imaging results.

\section{Image Acquisition}

Brain MRI was performed with a $3.0 \mathrm{~T}$ whole-body system (SignaTwinspeedHD.xt, GE Healthcare, Milwaukee, WI). Threedimensional, high-resolution anatomical images of the entire brain were obtained using an inversion-recovery prepared T1-weighted spoiled gradient echo pulse sequence $(\mathrm{TR}=25 \mathrm{~ms}, \mathrm{TE}=5 \mathrm{~ms}$, $\mathrm{TI}=$ $450 \mathrm{~ms}$, flip angle $=13^{\circ}$, field of view $=24 \mathrm{~cm}$, acquisition matrix $=$ $352 \times 224$, slice thickness $1.2 \mathrm{~mm}$, image resolution $0.47 \times 0.47 \mathrm{~mm})$ and an axial T2-weighted fast spin echo pulse sequence $(\mathrm{TR}=$ $6,660 \mathrm{~ms}, \mathrm{TE}=97.6 \mathrm{~ms}$, field of view $=24 \mathrm{~cm}$, acquisition matrix $=$ $512 \times 384$, slice thickness $3 \mathrm{~mm}$, image resolution $0.47 \times 0.47 \mathrm{~mm})$. An experienced neuroradiologist (IS) who was blinded to the participants' medical history evaluated all images regarding anatomical abnormalities and cerebral lesions. The 3D T1-weighted spoiled gradient-echo whole brain images were also used for morphometric assessment.

\section{Postprocessing}

Hippocampal segmentation was performed with FSL-FIRST (FSL; Oxford University, Oxford, UK $(40,41)$ ), a model based tool for segmentation and shape analysis, using the run_first_all command for the left and right hippocampus. This procedure is fully automated and includes a two-step registration to the MNI (Montreal Neurological Institute) template. Each hippocampus was then fit with a deformable surface mesh, using a Bayesian model based on training data from 336 participants including 153 children aged $4-16$ and 183 adults aged 16-87. The hippocampal volume was derived from the segmented images using fslstats-a software tool embedded in FSL (Figure 2). Total intracranial volume, and total grey matter, white matter, and cerebrospinal fluid volumes were also calculated for each participant with freesurfer, a standard morphometric analysis software (Harvard University, Cambridge, MA $(42,43))$. Both FSL and freesurfer are freely available software packages for noncommercial, scientific use. The test-retest reproducibility of the FIRST segmentation technique was assessed from repeated scans acquired within-session on three healthy adult volunteers, using the identical 3D T1-weighted spoiled gradient echo acquisition scan. Test-retest reproducibility was $3 \%$ for both the left and right hippocampi. We also performed a comparison between results derived from FSL-FIRST, and manual segmentations on 11 study participants and found a correlation coefficient of 0.8 ( $P$ $<0.001)$, which is in line with the literature on the technique (44).

\section{Statistics}

All behavioral and hippocampal volume data were analyzed using PASW statistics software (PASW (SPSS) statistics 19.0, IBM Corporation, New York, NY). Group comparisons between CHD patients and controls were done by two-sided $t$-tests and $\chi^{2}$ tests. To investigate positive associations between hippocampal outcome and functional (cognitive) outcome parameters, Spearman correlation analyses were performed independently for CHD patients and controls. These analyses were done excluding the CHD patients with overt brain lesions to rule out any possible confounding influence of the lesions-even though none of these lesions affected any structures close to the hippocampi or the hippocampal structures themselves. We then conducted linear regression analyses, each separately with one functional outcome variable as a dependent variable. Besides (relative) hippocampal volume, we entered age, and gender as independent variables to control for these possible confounders. These analyses were performed separately for patients and controls. We used $P$ values of 0.05 and 0.10 to enter or remove variables, respectively. 


\section{ACKNOWLEDGMENTS}

We thank Peter Klaver and Lars Michels for assessment of a subset of the control group, lanina Scheer for the anatomical evaluation of the images. Members of the Heart and Brain Research Group are: University Children's Hospital Zurich: Pediatric Neurology (Barbara R. Plecko), Pediatric Cardiology (Oliver Kretschmar, Walter Knirsch), Child Development Center (Beatrice Latal, Rabia Liamlahi, Michael von Rhein), Diagnostic Imaging (Christian Kellenberger, lanina Scheer), MR Center (Ruth O'Gorman Tuura), Pediatric Cardiac Surgery (Hitendu Dave), Anesthesiology (Christoph Bürki) and the University Hospital Lausanne, Congenital Cardiovascular Surgery (René Prêtre).

\section{STATEMENT OF FINANCIAL SUPPORT}

Swiss Heart Foundation, Else Kröner-Fresenius-Stiftung. P.P. was supported by US National Institutes of Health $(\mathrm{NIH})$ grant HD007446 through a Pediatric Academic Society-Society for Pediatric Research medical student grant. The sponsors had no influence on study design, the collection, analysis, and interpretation of data, the writing of the report, or the decision to submit the paper for publication. The manuscript has been written by the first and the last author.

Disclosure: No honorarium, grant, or other form of payment was given to anyone to produce the manuscript.

\section{REFERENCES}

1. Snookes SH, Gunn JK, Eldridge BJ, et al. A systematic review of motor and cognitive outcomes after early surgery for congenital heart disease. Pediatrics 2010;125:e818-27.

2. Bellinger DC, Wypij D, Rivkin MJ, et al. Adolescents with d-transposition of the great arteries corrected with the arterial switch procedure: neuropsychological assessment and structural brain imaging. Circulation 2011;124:1361-9.

3. Schaefer C, von Rhein M, Knirsch W, et al. Neurodevelopmental outcome, psychological adjustment, and quality of life in adolescents with congenital heart disease. Dev Med Child Neurol 2013;55:1143-9.

4. Bellinger DC, Rivkin MJ, DeMaso D, et al. Adolescents with tetralogy of Fallot: neuropsychological assessment and structural brain imaging. Cardiol Young 2015;25:338-47.

5. Miller SP, McQuillen PS, Hamrick S, et al. Abnormal brain development in newborns with congenital heart disease. N Engl J Med 2007;357:1928-38.

6. Licht DJ, Shera DM, Clancy RR, et al. Brain maturation is delayed in infants with complex congenital heart defects. J Thorac Cardiovasc Surg 2009; 137:529-36; discussion 536-7.

7. Limperopoulos C, Tworetzky W, McElhinney DB, et al. Brain volume and metabolism in fetuses with congenital heart disease: evaluation with quantitative magnetic resonance imaging and spectroscopy. Circulation 2010;121:26-33.

8. Ortinau C, Inder T, Lambeth J, Wallendorf M, Finucane K, Beca J. Congenital heart disease affects cerebral size but not brain growth. Pediatr Cardiol 2012;33:1138-46.

9. von Rhein M, Buchmann A, Hagmann C, et al. Severe congenital heart defects are associated with global reduction of neonatal brain volumes. J Pediatr 2015;167:1259-63.e1.

10. von Rhein M, Buchmann A, Hagmann C, et al. Brain volumes predict neurodevelopment in adolescents after surgery for congenital heart disease. Brain 2014;137(Pt 1):268-76.

11. Rollins CK, Watson CG, Asaro LA, et al. White matter microstructure and cognition in adolescents with congenital heart disease. J Pediatr 2014;165:936-44.e1-2.

12. Nadel L, Peterson MA. The hippocampus: part of an interactive posterior representational system spanning perceptual and memorial systems. J Exp Psychol Gen 2013;142:1242-54.

13. Beauchamp MH, Thompson DK, Howard K, et al. Preterm infant hippocampal volumes correlate with later working memory deficits. Brain 2008;131(Pt 11):2986-94.

14. Isaacs EB, Lucas A, Chong WK, et al. Hippocampal volume and everyday memory in children of very low birth weight. Pediatr Res 2000;47:713-20.
15. von Rhein M, Scheer I, Loenneker T, Huber R, Knirsch W, Latal B. Structural brain lesions in adolescents with congenital heart disease. J Pediatr 2011;158:984-9.

16. von Rhein M, Kugler J, Liamlahi R, Knirsch W, Latal B, Kaufmann L. Persistence of visuo-constructional and executive deficits in adolescents after open-heart surgery. Res Dev Disabil 2014;36C:303-10.

17. DeMaso DR, Labella M, Taylor GA, et al. Psychiatric disorders and function in adolescents with d-transposition of the great arteries. J Pediatr 2014;165:760-6.

18. Cassidy AR, White MT, DeMaso DR, Newburger JW, Bellinger DC. Executive function in children and adolescents with critical cyanotic congenital heart disease. J Int Neuropsychol Soc 2014:1-16.

19. Saigal S, Doyle LW. An overview of mortality and sequelae of preterm birth from infancy to adulthood. Lancet 2008;371:261-9.

20. Taylor HG, Filipek PA, Juranek J, Bangert B, Minich N, Hack M. Brain volumes in adolescents with very low birth weight: effects on brain structure and associations with neuropsychological outcomes. Dev Neuropsychol 2011;36:96-117.

21. Martinussen M, Flanders DW, Fischl B, et al. Segmental brain volumes and cognitive and perceptual correlates in 15-year-old adolescents with low birth weight. J Pediatr 2009;155:848-53 e1.

22. Peterson BS, Vohr B, Staib LH, et al. Regional brain volume abnormalities and long-term cognitive outcome in preterm infants. JAMA 2000;284:1939-47.

23. McQuillen PS, Miller SP. Congenital heart disease and brain development. Ann N Y Acad Sci 2010;1184:68-86.

24. Shohamy D, Turk-Browne NB. Mechanisms for widespread hippocampal involvement in cognition. J Exp Psychol Gen 2013;142:1159-70.

25. Oechslin MS, Descloux C, Croquelois A, et al. Hippocampal volume predicts fluid intelligence in musically trained people. Hippocampus 2013;23:552-8

26. Piekema C, Kessels RP, Mars RB, Petersson KM, Fernández G. The right hippocampus participates in short-term memory maintenance of objectlocation associations. Neuroimage 2006;33:374-82.

27. Ranganath C. Working memory for visual objects: complementary roles of inferior temporal, medial temporal, and prefrontal cortex. Neuroscience 2006;139:277-89.

28. Ranganath C, Cohen MX, Dam C, D'Esposito M. Inferior temporal, prefrontal, and hippocampal contributions to visual working memory maintenance and associative memory retrieval. J Neurosci 2004;24:3917-25.

29. Opitz B. Memory function and the hippocampus. Front Neurol Neurosci 2014;34:51-9.

30. Eichenbaum H, Cohen NJ. Can we reconcile the declarative memory and spatial navigation views on hippocampal function? Neuron 2014;83: 764-70.

31. Harms MP, Wang L, Csernansky JG, Barch DM. Structure-function relationship of working memory activity with hippocampal and prefrontal cortex volumes. Brain Struct Funct 2013;218:173-86.

32. Axmacher N, Mormann F, Fernández G, Cohen MX, Elger CE, Fell J. Sustained neural activity patterns during working memory in the human medial temporal lobe. J Neurosci 2007;27:7807-16.

33. Ranganath C, D'Esposito M. Medial temporal lobe activity associated with active maintenance of novel information. Neuron 2001;31:865-73.

34. Lan WC, Priestley M, Mayoral SR, Tian L, Shamloo M, Penn AA. Sexspecific cognitive deficits and regional brain volume loss in mice exposed to chronic, sublethal hypoxia. Pediatr Res 2011;70:15-20.

35. Roth TC 2nd, Brodin A, Smulders TV, LaDage LD, Pravosudov VV. Is bigger always better? A critical appraisal of the use of volumetric analysis in the study of the hippocampus. Philos Trans R Soc Lond B Biol Sci 2010;365:915-31.

36. Van Petten C. Relationship between hippocampal volume and memory ability in healthy individuals across the lifespan: review and meta-analysis. Neuropsychologia 2004;42:1394-413.

37. Gaynor JW, Stopp C, Wypij D, et al.; International Cardiac Collaborative on Neurodevelopment (ICCON) Investigators. Neurodevelop- 
mental outcomes after cardiac surgery in infancy. Pediatrics 2015;135: 816-25.

38. Rogers CE, Anderson PJ, Thompson DK, et al. Regional cerebral development at term relates to school-age social-emotional development in very preterm children. J Am Acad Child Adolesc Psychiatry 2012;51:181-91.

39. Landolt MA, Valsangiacomo Buechel ER, Latal B. Health-related quality of life in children and adolescents after open-heart surgery. J Pediatr 2008;152:349-55.

40. Patenaude B, Smith SM, Kennedy DN, Jenkinson M. A Bayesian model of shape and appearance for subcortical brain segmentation. Neuroimage 2011;56:907-22.
41. Jenkinson M, Beckmann CF, Behrens TE, Woolrich MW, Smith SM. FSL. Neuroimage 2012;62:782-90.

42. Dale AM, Fischl B, Sereno MI. Cortical surface-based analysis. I. Segmentation and surface reconstruction. Neuroimage 1999;9:179-94.

43. Fischl B, Salat DH, Busa E, et al. Whole brain segmentation: automated labeling of neuroanatomical structures in the human brain. Neuron 2002;33:341-55.

44. Nugent AC, Luckenbaugh DA, Wood SE, Bogers W, Zarate CA Jr, Drevets WC. Automated subcortical segmentation using FIRST: test-retest reliability, interscanner reliability, and comparison to manual segmentation. Hum Brain Mapp 2013;34:2313-29. 